\title{
Effect of Periodicity of Exercise on Serum Metabolites of Stall Housed competition Horses under Climatic Conditions of Odisha, India
}

\author{
S. Kanungo ${ }^{1 *}$, C.R. Pradhan ${ }^{1}$, L.K. Babu ${ }^{1}$, K. Behera ${ }^{1}$, A.K. Palei ${ }^{1}$, \\ B. Jena ${ }^{2}$ and D.P. Das ${ }^{3}$ \\ ${ }^{1}$ Department of Livestock Production Management, ${ }^{2}$ Department of Argo, \\ ${ }^{3}$ Department of Pathology, C.V.Sc. and A. H., O.U.A.T., Bhubaneswar-751003, India \\ *Corresponding author
}

\begin{abstract}
A B S T R A C T
Keywords

Exercise,

Hematological, Serum

biochemical,

Parameters, Horses,

Hot climate

Article Info

Accepted:

26 February 2018

Available Online:

10 March 2018

The present study was carried out in the thoroughbred stall housed horses maintained at College of Veterinary Science and Animal Husbandry, Bhubaneswar under hot and humid climatic conditions of Odisha with an objective to determine the effect of exercise on hematological and serum biochemical indices. Blood samples were drawn from jugular veins of the animals in the morning and 30, 240 and 480 minutes after exercise and subsequently assessed for haematological and serum biochemical parameters. It was confirmed that the mean total erythrocyte and leucocyte, haemoglobin concentration, Packed Cell Volume, total serum protein values increased immediately after the exercise, whereas the ESR, whole blood glucose showed decreasing trends. Further, the serum chloride level decreased significantly 4 hours after the exercise, but the values related to serum $\mathrm{Na}, \mathrm{K}, \mathrm{Mg}, \mathrm{Ca}$ and $\mathrm{P}$ levels were not statistically significant after exercise in the horses. It can be concluded that the horses maintained under hot and humid climatic conditions of Odisha exhibited similar changes in blood when given exercise and the changes were of transitory nature. Further study is needed to be taken up to ascertain the facts responsible for a low erythrocyte count in these horses.
\end{abstract}

\section{Introduction}

Variations in hematological parameters in horses are associated with several factors such as exercise and training, feeding, age, sex, breed, diurnal and seasonal variation, temperature and the physiological status etc. Physical, hematological, and biochemical changes associated with exercise have been extensively analyzed in several types of horses such as Thoroughbreds (Mukai et al., 2007), endurance horses (Santos et al., 2001 and
Teixiera-Neto et al., 2008 and Munoz et al., 2006), and show jumpers (Aguilera et al., 2000). The performance of the athlete is determined by many complicated interdependent haematochemical and physiological processes (Warwick, 2004). According to Lindinger and Heingenhauser (2008), other parameters can be used to determine the effect of exercise, such as glucose, enzymatic and haematochemical parameters, and electrolytes $\left(\mathrm{Na}^{+}, \mathrm{K}^{+}\right.$and $\left.\mathrm{Cl}^{-}\right)$ with the purpose of defining reliable 
parameters for the horse's performance assessment. In the horse, electrolytes play an important role in maintaining osmotic pressure, fluid balance, and nerve and muscle activity (Frape, 2010). So, it is important to have some idea of the magnitude of loss of electrolytes from a horse during exercise (Van den berg, 2009). Since it is important to analyse the modifications of these parameters in the function of different systems and types of energy utilized (De Miranda et al., 2009), haematological, biochemical and electrolytic parameters have largely been evaluated during different kinds of physical effort, such as trot races (Tateo et al., 2008 and Piccione et al., 2009) and endurance training and racing (Lindinger and Heingenhauser, 2008; Robert et al., 2010; Munoz et al., 2010 The climatic conditions of Odisha differ a lot from that of other parts of country. There is more rain and this causes increased humidity. A fair idea of hematological changes in these animals is necessary to show as to how they behave in Odisha climate, after work stress, under different climatic conditions. So, an effort was made to study the haematological and serum biochemical variations correlated with performance in the Jumper horses before and after exercise to know their athletic potentials thus addressing a paucity of scientific data in this area. Further, it was hoped that this work would provide a foundation to develop a regime that can be used for screening potential of competition horses.

\section{Materials and Methods}

The present study was carried out in the thoroughbred stall housed horses maintained at College of Veterinary Science and Animal Husbandry, Bhubaneswar under hot and humid climatic conditions of Odisha with an objective to determine the effect of exercise on hematological and serum biochemical indices. Four gelds and two mares within the age group of five to fourteen years on balanced diet were selected and were regularly put to exercise six days per week. However, before the animals were used for experiments, routine checkup of faecal samples were done. The horses were vaccinated against Anthrax and Mallein test was conducted as a regular routine. Four gelds and two mares were used as the control group (Group-I) in order to record the normal hematological and serum biochemical parameters such as total erythrocyte (RBC), total leucocytes (WBC), Haemoglobin (Hb), Packed Cell volume (PCV), Mean Corpuscular Volume (MCV), Mean Corpuscular Hemoglobin (MCH), Mean Corpuscular Hemoglobin Concentration (MCHC), Erythrocyte Sedimentation Rate (ESR), Serum total glucose, Whole blood glucose, Serum Sodium, Potassium, Calcium, Magnesium and Chloride levels were estimated in the early morning at 06.30AM. Subsequently, these parameters were recorded in the same horses immediately after putting them to exercise for 30 minutes (Group-II), one hundred forty minutes (4 hours) after exercise (Group-III) and four hundred eighty minutes ( 8 hours) after the exercise (GroupIV) respectively.

\section{Collection and preservation of blood and serum}

Five milliliters of blood were collected at 05.40. hrs by jugular venipuncture into evacuated collection tubes. For haematological study blood was collected in sterile vial using EDTA as anticoagulant @ 1 $\mathrm{mg} / \mathrm{ml}$ of blood as recommended by Jain (1986) and for collection and preservation of serum $10 \mathrm{ml}$ of blood was collected from jugular vein of each horse in sterilized test tubes. The tubes containing blood were kept in slanting position and the blood was allowed to clot. After the blood got clotted, the tubes were transferred to refrigerator at 4 degree centigrade for 12 hours to allow maximum secretion of serum from the clot. Then the 
serum was pipetted out from the tubes and was centrifuged at 2,500 rpm for 5 minutes to separate unlysed cells and other darts. The clear serum was collected carefully into sterilized vials and stored in the frozen chamber of the refrigerator. No preservatives were added to the serum. Before using the frozen serum for experiment, it was allowed to defrost at room temperature.

\section{Estimation haematological and serum biochemical parameters}

Sahli's acid hematin method was employed for estimation of haemoglobin by using N/10 Hydrochloric acid $(\mathrm{HCl})$ and expressed as $\mathrm{g} / \mathrm{dl}$ (Coles, 1986). The PCV (\%) and TEC (no. of erythrocytes $\times 10^{6} / \mu$ l of blood) were estimated by Wintrob's haematocrit method and haemocytometer method respectively as described by Coles (1986). Similarily, the TLC (no.of leucocytes $\times 10^{3} / \mu$ l of blood) and $\operatorname{ESR}(\mathrm{mm} / / \mathrm{hr}$ fall $)$ were estimated by haemocytometer method and the procedure described by Coles (1986) respectively. The glucose levels [milligrams per decilitre $(\mathrm{mg} / \mathrm{dL}) / \mathrm{m}(\%)]$ and total serum protein [grams per deciliter $(\mathrm{g} / \mathrm{dL})]$ were estimated by Modified International Federation of Clinical Chemistry and Laboratory Medicine (IFCC) method (Burtis and Ashwood, 1999) and Biuret method (Johnson et al., 1999). respectively by using the diagnostic kits supplied by M/s Crest Biosystem ${ }^{\mathrm{TM}}$, a division of Coral clinical systems, Goa. Further, the concentration of serum calcium (mg/dl), phosphorous (mg/dl), magnesium (mg/dl), sodium $(\mathrm{mg} / \mathrm{dl})$ and potassium $(\mathrm{mg} / \mathrm{dl})$ were estimated by Modified International Federation of Clinical Chemistry and Laboratory Medicine (IFCC) method as per the procedure described by Burtis and Ashwood (1999), using the reagent kit supplied by Crest Biosystems ${ }^{\mathrm{TM}}$, a division of Coral clinical systems, Goa.
All behavior and weather parameter data were averaged to obtain values for statistical analysis. Rectal temperature (RT), Respiration Rate (RR) and Pulse Rate (PR) were measured and analyzed. Different behavior of horse was recorded for last 15 days in each month for analysis. The results of the study were recorded and statistically analyzed as per the methods suggested by Snedecor Cochran and (1989).

\section{Results and Discussion}

The mean values of the hematological parameters of six horses before exercise have been enlisted in Table 1 and values immediately after exercise are shown in Table 2. The respective values at 240 minutes and 480 minutes following exercise are given in Table 3 and 4 . The mean values of all the parameters indicating degree of significance have been shown in Table 5 .

The mean total erythrocyte concentration in the horses of Group I was estimated to be 5.28 $\pm 0.44 \times 10^{6} \mathrm{cmm}$. Immediately after exercise the value was $6.73 \pm 0.43 \times 10^{6} \mathrm{cmm}$. Analysis of variance showed significant $(\mathrm{P} \leq$ 0.05) Increase in total erythrocyte concentration immediately after exercise. Critical difference also indicated a significant $(\mathrm{P} \leq 0.05)$ increase. Subsequent estimations of total erythrocyte values at 240 minutes and 480 minutes after exercise were not found to be significant.

The mean total leucocyte value of the experimental animals at rest in Group I was evaluated to be $9708.33 \pm 352.40 \mathrm{cmm}$. There was a rise in the leucocyte level in Group II and III. However, this was not statistically significant. The level of total leucocytes at 480 minutes after exercise was though less than the control group and was not significant (Table 5). 
The mean hemoglobin level of the experimental horses in control group was recorded to be $10.33 \pm 0.63$ gram Percent. Analysis of variance showed significant $(\mathrm{P} \leq$ 0.01 ) increase just after exercise. Immediately after exercise the horses (Group II) showed an increase in hemoglobin level to $11.83 \pm 0.54$ g. percent and this was statistically signification of hemoglobin level were found to be not significant (Table 5).

The mean packed cell volume of the control group of horses (Group I) was found to be $29.83 \pm 1.19$ percent. Analysis of variance indicated significant $(p \leq 0.01)$ increase immediately after exercise. The mean packed cell volume immediately after exercise was estimated to be $32.83 \pm 1.35$ percent. This was significantly $(\mathrm{p} \leq 0.05)$ high in comparison to group I. The mean values of this parameter at 240 and 480 minutes after exercise were not significant (Table 5).

The mean corpuscular volume of the horses in group I was calculated to be $57.84 \pm 3.61 \mathrm{cu} \mu$. Following exercise estimation of the mean corpuscular volume at different intervals was found to be not significant with regard to the control group (Table 5).

The mean corpuscular hemoglobin level of the horses in the control group was assessed to be $20.13 \pm 1.66 \mu \mu \mathrm{g}$. Estimation of this parameter at different intervals after exercise was found to be not significant (Table 5).

The mean corpuscular hemoglobin concentration of the horses in Group I was calculated to be $34.63 \pm 1.54$ percent. The mean value of this parameter following exercise at different interval of time was found to be not significant (table 5).

The mean erythrocyte sedimentation rate of the experimental horses in control group was found to be $34.17 \pm .0 .75$ millimeter. The analysis of variance showed that following exercise there was a significant $(\mathrm{p} \leq 0.01)$ decrease after exercise. The mean level of this parameter immediately (Group II) after exercise was recorded to be $10.33 \pm 1.2$ and $25.67 \pm 1.14$ millimeter respectively. The mean values of erythrocyte sedimentation rate as stated above after exercise were not significant within themselves and also in comparison with the control group. However, the mean level of the parameter 480 minutes after exercise was not significant with regard to the control group (Table 5).

The mean level of serum total protein in the horses at rest was estimate to be $7.45 \pm 0.14$ gm\%. Subsequent estimation after exercise showed significant $(\mathrm{p} \leq 0.01)$ increase. Immediately following exercise the mean level of serum total protein was recorded to be $8.45 \pm 0.26 \mathrm{~g}$. percent and was significantly ( $\mathrm{p}$ $\leq 0.05$ ) higher than the control group. Two hundred and forty minutes (Group-III) after exercise the mean level dropped to $7.87 \leq 0.27$ gram percent and was not significant in comparison to the levels in the horses of group I and Group II. However, the mean value of this parameter in the horses, 480 minutes after exercise (Group IV) was found to be less than the values of Group II and Group III (Table 5).

The mean level of whole blood glucose in group I (Control) was estimated to be 80.00 $+ \pm 3.87 \mathrm{mg}$. percent. Subsequent estimations after exercise was found to be significantly ( $\mathrm{P}$ $\leq 0$. 01) low. The mean level in group II was $71.67 \pm 4.62 \mathrm{mg}$. percent and was significantly $(\mathrm{P} \leq 0.05)$ low. The glucose level was found to be $77.00 \leq 4.09 \mathrm{mg}$. percent in the horse of Group III and this was not significant with regard to the values of Group I and Group II. The mean glucose level of the horses in Group II. 
Table.1 Hematological parameters of horse before exercise (Group I)

\begin{tabular}{|c|c|c|c|c|c|c|c|c|c|c|c|c|c|c|c|}
\hline $\begin{array}{l}\text { Hors } \\
\text { e No. }\end{array}$ & $\begin{array}{l}\text { RBC } \\
(\mathrm{Cmm})\end{array}$ & $\begin{array}{l}\text { WBC } \\
(\mathrm{Cmm})\end{array}$ & $\begin{array}{l}\text { Hb } \\
(\mathrm{g} \% \\
)\end{array}$ & $\begin{array}{l}\text { PCV } \\
(\%)\end{array}$ & $\begin{array}{l}\text { MCV } \\
\text { сuн }\end{array}$ & $\begin{array}{l}\text { MC } \\
\mathbf{H} \\
\text { (ppg) }\end{array}$ & $\begin{array}{l}\text { MCH } \\
\text { C } \\
\text { percen } \\
\text { t }\end{array}$ & $\begin{array}{l}\text { ESR } \\
\mathrm{mm} / 3 \\
0 \\
\text { mins }\end{array}$ & $\begin{array}{l}\text { Total } \\
\text { protei } \\
\text { n } \\
(\mathrm{g} \%)\end{array}$ & $\begin{array}{l}\text { Glucos } \\
\text { e } \\
(\mathrm{mg} \%)\end{array}$ & $\begin{array}{l}\mathrm{Na} \\
\mathrm{m}^{\mathrm{E}} \mathrm{q} / \\
\mathrm{L}\end{array}$ & $\begin{array}{l}\mathrm{K} \\
\mathbf{m}^{\mathrm{E}} \mathbf{q} / \\
\mathbf{L}\end{array}$ & $\begin{array}{l}\mathrm{Ca} \\
\left(\mathrm{mg}^{\%}\right.\end{array}$ & $\begin{array}{l}\mathrm{Mg}_{2} \\
\mathrm{~m}^{\mathrm{E}} \mathbf{q} / \\
\mathrm{L}\end{array}$ & $\begin{array}{l}\mathrm{Cl}_{2} \\
\mathrm{~m}^{\mathrm{E}} \mathbf{q} / \\
\mathrm{L}\end{array}$ \\
\hline 1 & $6.29 \times 10^{6}$ & 8.250 & 8.5 & 28 & 13.50 & 44.51 & 30.35 & 36 & 7.50 & 80 & 144 & 4.571 & 12.00 & 2.80 & 102 \\
\hline 2 & $5.89 \times 10^{6}$ & 10.050 & 13 & 32 & $\begin{array}{l}22.07 \\
1\end{array}$ & 54.30 & 40.62 & 35 & 7.75 & 75 & 156 & 4.428 & 11.30 & 2.80 & 82 \\
\hline 3 & $4.12 \times 10^{6}$ & 10.400 & 10 & 27 & $\begin{array}{l}24.27 \\
1\end{array}$ & 65.50 & 37.03 & 32 & 7.20 & 80 & 140 & 4.285 & 13.10 & 2.90 & 106 \\
\hline 4 & $6.49 \times 10^{6}$ & 10.600 & 11 & 34 & $\begin{array}{l}16.94 \\
9\end{array}$ & 52.30 & 32.35 & 36 & 6.85 & 90 & 135 & 4.928 & 12.30 & 2.65 & 128 \\
\hline 5 & $4.65 \times 10^{6}$ & 9.250 & 10 & 31 & $\begin{array}{l}21.50 \\
5\end{array}$ & 66.60 & 32.25 & 34 & 7.70 & 65 & 148 & 4.50 & 11.90 & 2.75 & 112 \\
\hline 6 & $4.23 \times 10^{6}$ & 9.700 & 9.5 & 27 & $\begin{array}{l}22.45 \\
8\end{array}$ & 63.80 & 35.18 & 32 & 7.70 & 90 & 142 & 4.571 & 12.00 & 2.80 & 102 \\
\hline $\begin{array}{l}\text { Mea } \\
\text { n } \\
\pm \text { S.E. }\end{array}$ & $\begin{array}{l}5.28 \times 10^{6} \\
\pm 0.44 \times 10\end{array}$ & $\begin{array}{l}9,708.3 \\
3 \\
\pm 352.4 \\
0\end{array}$ & $\begin{array}{l}10.3 \\
3 \\
\pm 0.6 \\
3\end{array}$ & $\begin{array}{l}29.8 \\
3 \\
\pm 1.1 \\
9\end{array}$ & $\begin{array}{l}57.84 \\
\pm 3.61\end{array}$ & $\begin{array}{l}20.13 \\
\pm 1.66\end{array}$ & $\begin{array}{l}34.63 \\
\pm 1.54\end{array}$ & $\begin{array}{l}34.17 \\
\pm 0.75\end{array}$ & $\begin{array}{l}7.45 \\
\pm 0.147\end{array}$ & $\begin{array}{l}80.00 \\
\pm 3.87\end{array}$ & $\begin{array}{l}144.1 \\
7 \\
\pm 2.95\end{array}$ & $\begin{array}{l}4.547 \\
\pm 0.08 \\
8\end{array}$ & $\begin{array}{l}12.10 \\
\pm 0.24 \\
1\end{array}$ & $\begin{array}{l}2.78 \\
\pm 0.03 \\
3\end{array}$ & $\begin{array}{l}105.3 \\
3 \\
\pm 6.12\end{array}$ \\
\hline
\end{tabular}

Table.2 Hematological parameters of horse after exercise (Group II)

\begin{tabular}{|c|c|c|c|c|c|c|c|c|c|c|c|c|c|c|c|}
\hline $\begin{array}{l}\text { Horse } \\
\text { No. }\end{array}$ & $\begin{array}{l}\text { RBC } \\
(\mathbf{C m m})\end{array}$ & $\begin{array}{l}\text { WBC } \\
(\mathrm{Cmm})\end{array}$ & $\begin{array}{l}\mathrm{Hb} \\
(\mathrm{g} \%)\end{array}$ & $\begin{array}{l}\text { PCV } \\
(\%)\end{array}$ & $\begin{array}{l}\text { MCV } \\
\text { сu } \mu\end{array}$ & $\begin{array}{l}\text { MCH } \\
\text { (ppg) }\end{array}$ & $\begin{array}{l}\text { MCHC } \\
\text { percent }\end{array}$ & $\begin{array}{l}\text { ESR } \\
\mathrm{mm} / 30 \\
\mathrm{mins}\end{array}$ & $\begin{array}{l}\text { Total } \\
\text { protein } \\
(\mathrm{g} \%)\end{array}$ & $\begin{array}{l}\text { Glucose } \\
(\mathrm{mg} \%)\end{array}$ & $\begin{array}{l}\mathrm{Na} \\
\mathrm{m}^{\mathrm{E}} q / \mathrm{L}\end{array}$ & $\begin{array}{l}\mathrm{K} \\
\mathrm{m}^{\mathrm{E}} \mathrm{q} / \mathrm{L}\end{array}$ & $\begin{array}{l}\mathrm{Ca} \\
\left(\mathrm{mg}^{\%}\right)\end{array}$ & $\begin{array}{l}\mathrm{Mg}_{2} \\
\mathrm{~m}^{\mathrm{E}} \mathrm{q} / \mathrm{L}\end{array}$ & $\begin{array}{l}\mathrm{Cl}_{2} \\
\mathrm{~m}^{\mathrm{E}} \mathrm{q} / \mathrm{L}\end{array}$ \\
\hline 1 & $7.89 \times 10^{6}$ & 13,000 & 10.00 & 29 & 36.75 & 12.67 & 34.48 & 11 & 8 & 78 & 145 & 5.00 & 11.9 & 2.75 & 116 \\
\hline 2 & $6.74 \times 10^{6}$ & 11,850 & 14.00 & 37 & 54.80 & 20.771 & 37.83 & 9 & 9.25 & 70 & 152 & 4.928 & 11 & 2.80 & 74 \\
\hline 3 & $5.56 \times 10^{6}$ & 12,350 & 11.50 & 30 & 53.90 & 20.682 & 38.33 & 7 & 8.25 & 82 & 142 & 3.285 & 13 & 2.90 & 100 \\
\hline 4 & $8.10 \times 10^{6}$ & 12,050 & 12.50 & 36 & 44.40 & 15.432 & 34.72 & 12 & 7.70 & 75 & 154 & 4.857 & 12 & 2.90 & 125 \\
\hline 5 & $6.13 \times 10^{6}$ & 10,800 & 11.50 & 34 & 55.40 & 18.76 & 33.82 & 15 & 8.25 & 50 & 144 & 4.50 & 11.6 & 2.75 & 98 \\
\hline 6 & $5.95 \times 10^{6}$ & 10,300 & 11.50 & 31 & 52.10 & 19.327 & 37.09 & 8 & 9.25 & 75 & 158 & 4.50 & 13 & 2.80 & 100 \\
\hline $\begin{array}{l}\text { Mean } \\
\pm \text { S.E. }\end{array}$ & $\begin{array}{l}6.73 \times 10^{6} \\
\pm 0.43 \times 10^{6}\end{array}$ & $\begin{array}{l}11.725 .00 \\
\pm 409.54\end{array}$ & $\begin{array}{l}11.83 \\
\pm 0.54\end{array}$ & $\begin{array}{l}32.82 \\
\pm 1.35\end{array}$ & $\begin{array}{l}49.55 \\
\pm 3.04\end{array}$ & $\begin{array}{l}17.94 \\
\pm 1.32\end{array}$ & $\begin{array}{l}36.05 \\
\pm 0.79\end{array}$ & $\begin{array}{l}10.33 \\
\pm 1.20\end{array}$ & $\begin{array}{l}8.45 \pm \\
0.265\end{array}$ & $\begin{array}{l}71.67 \pm \\
4.62\end{array}$ & $\begin{array}{l}149.17 \pm \\
2.61\end{array}$ & $\begin{array}{l}4.51 \pm \\
0.261\end{array}$ & $\begin{array}{l}12.08 \pm \\
0.32\end{array}$ & $\begin{array}{l}2.80 \pm \\
0.02\end{array}$ & $\begin{array}{l}102.17 \\
\pm 7.15\end{array}$ \\
\hline
\end{tabular}

Table.3 Hematological parameters of horse 240 minutes (4 hours) after exercise (Group III)

\begin{tabular}{|c|c|c|c|c|c|c|c|c|c|c|c|c|c|c|c|}
\hline $\begin{array}{l}\text { Horse } \\
\text { No. }\end{array}$ & $\begin{array}{l}\text { RBC } \\
(\mathbf{C m m})\end{array}$ & $\begin{array}{l}\text { WBC } \\
(\mathbf{C m m})\end{array}$ & $\begin{array}{l}\text { Hb } \\
(\mathrm{g} \%)\end{array}$ & $\begin{array}{l}\text { PCV } \\
(\%)\end{array}$ & $\begin{array}{l}\text { MCV } \\
\text { сu} \mu\end{array}$ & $\begin{array}{l}\text { MCH } \\
\text { (ppg) }\end{array}$ & $\begin{array}{l}\text { MCHC } \\
\text { percent }\end{array}$ & $\begin{array}{l}\text { ESR } \\
\mathrm{mm} / 30 \\
\mathrm{mins}\end{array}$ & $\begin{array}{l}\text { Total } \\
\text { protein } \\
(\mathbf{g} \%)\end{array}$ & $\begin{array}{l}\text { Glucose } \\
\text { (mg\%) }\end{array}$ & $\begin{array}{l}\mathrm{Na} \\
\mathrm{m}^{\mathrm{E}} \mathrm{q} / \mathrm{L}\end{array}$ & $\begin{array}{l}\mathrm{K} \\
\mathrm{m}^{\mathrm{E}} \mathrm{q} / \mathrm{L}\end{array}$ & $\begin{array}{l}\mathrm{Ca} \\
\left(\mathrm{mg}{ }^{\%}\right)\end{array}$ & $\begin{array}{l}\mathrm{Mg}_{2} \\
\mathrm{~m}^{\mathrm{E}} \mathrm{q} / \mathrm{L}\end{array}$ & $\begin{array}{l}\mathrm{Cl}_{2} \\
\mathrm{~m}^{\mathrm{E}} \mathrm{q} / \mathrm{L}\end{array}$ \\
\hline 1 & $5.44 \times 10^{6}$ & 8.400 & 8.50 & 26 & 47.70 & 15.625 & 32.69 & 25 & 7.70 & 84 & 144 & 4.285 & 10.40 & 2.75 & 92 \\
\hline 2 & $4.55 \times 10^{6}$ & 12.600 & 13.00 & 29 & 63.70 & 28.571 & 44.82 & 23 & 8.25 & 70 & 160 & 4.87 & 12.70 & 2.80 & 80 \\
\hline 3 & $5.58 \times 10^{6}$ & 8.850 & 10.00 & 27 & 48.30 & 17.921 & 37.03 & 25 & 7.20 & 90 & 142 & 3.785 & 11 & 2.80 & 94 \\
\hline 4 & $6.57 \times 10^{6}$ & 10.500 & 11.5 & 35 & 53.20 & 17.503 & 32.85 & 26 & 9.00 & 80 & 150 & 4.352 & 13.70 & 2.65 & 118 \\
\hline 5 & $8.03 \times 10^{6}$ & 8.500 & 11.00 & 32 & 39.80 & 13.698 & 34.37 & 31 & 7.33 & 62 & 130 & 3.571 & 13.20 & 2.75 & 95 \\
\hline 6 & $4.35 \times 10^{6}$ & 10.150 & 9.5 & 27 & 62.06 & 21.839 & 35.18 & 24 & 7.70 & 76 & 130 & 4.50 & 14.30 & 2.75 & 102 \\
\hline $\begin{array}{l}\text { Mean: } \\
\text { S.E. }\end{array}$ & $\begin{array}{l}5.75 \times 10^{6} \\
\pm 0.56 \times 10^{6}\end{array}$ & $\begin{array}{l}9.833 .33 \\
\pm 658.10\end{array}$ & $\begin{array}{l}10.58 \pm \\
0.65\end{array}$ & $\begin{array}{l}29.33 \pm \\
1.43\end{array}$ & $\begin{array}{l}52.46 \pm \\
1.29\end{array}$ & $\begin{array}{l}19.19 \pm \\
2.18\end{array}$ & $\begin{array}{l}36.16 \pm \\
1.85\end{array}$ & $\begin{array}{l}25.67 \pm \\
1.14\end{array}$ & $\begin{array}{l}7.87 \pm \\
0.27\end{array}$ & $\begin{array}{l}77 \pm \\
4.09\end{array}$ & $\begin{array}{l}142.67 \\
\pm 4.75\end{array}$ & $\begin{array}{l}4.33 \pm \\
0.196\end{array}$ & $\begin{array}{l}12.55 \pm \\
0.628\end{array}$ & $\begin{array}{l}2.75 \pm \\
0.02\end{array}$ & $\begin{array}{l}96.83 \\
\pm 5.14\end{array}$ \\
\hline
\end{tabular}

Table.4 Hematological parameters of horse 480 minutes ( 8 hours) after exercise (Group IV)

\begin{tabular}{|c|c|c|c|c|c|c|c|c|c|c|c|c|c|c|c|}
\hline $\begin{array}{l}\text { Horse } \\
\text { No. }\end{array}$ & $\begin{array}{l}\text { RBC } \\
(\mathbf{C m m})\end{array}$ & $\begin{array}{l}\text { WBC } \\
(\mathbf{C m m})\end{array}$ & $\begin{array}{l}\mathrm{Hb} \\
(\mathrm{g} \%)\end{array}$ & $\begin{array}{l}\text { PCV } \\
(\%)\end{array}$ & 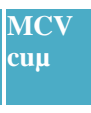 & $\begin{array}{l}\text { MCH } \\
(p p g)\end{array}$ & $\begin{array}{l}\text { MCHC } \\
\text { percent }\end{array}$ & $\begin{array}{l}\text { ESR } \\
\mathrm{mm} / \mathbf{3 0} \\
\mathrm{mins}\end{array}$ & $\begin{array}{l}\text { Total } \\
\text { protein } \\
(\mathrm{g} \%)\end{array}$ & $\begin{array}{l}\text { Glucose } \\
(\mathrm{mg} \%)\end{array}$ & $\begin{array}{l}\mathrm{Na} \\
\mathrm{m}^{\mathrm{E}} \mathrm{q} / \mathrm{L}\end{array}$ & $\mathrm{m}^{\mathrm{E}} \mathbf{q} / \mathrm{L}$ & $\begin{array}{l}\mathrm{Ca} \\
(\mathrm{mg} \%)\end{array}$ & $\mid \begin{array}{l}\mathrm{Mg}_{2} \\
\mathrm{~m}^{\mathrm{E}} \mathbf{q} / \mathrm{L}\end{array}$ & $\begin{array}{l}\mathrm{Cl}_{2} \\
\mathrm{~m}^{\mathrm{E}} \mathrm{q} / \mathrm{L}\end{array}$ \\
\hline 1 & $5.65 \times 10^{6}$ & 8.300 & 9.00 & 26 & 46.00 & 15.929 & 34.69 & 34 & 7.65 & 82 & 130 & 3.857 & 11.40 & 2.75 & 103 \\
\hline 2 & $5.35 \times 10^{6}$ & 11.300 & 13.00 & 32 & 59.70 & 24.299 & 40.62 & 34 & 7.80 & 90 & 142 & 4.571 & 12.90 & 2.80 & 82 \\
\hline 3 & $4.33 \times 10^{6}$ & 8.550 & 10.00 & 27 & 62.30 & 23.094 & 37.03 & 32 & 7.20 & 96 & 125 & 3.857 & 12.30 & 2.80 & 103 \\
\hline 4 & $5.72 \times 10^{6}$ & 8.600 & 11.00 & 34 & 59.50 & 19.23 & 32.35 & 37 & 6.15 & 86 & 156 & 4.87 & 12.40 & 2.65 & 130 \\
\hline 5 & $675 \times 10^{6}$ & 9.900 & 10.00 & 31 & 45.90 & 14.814 & 32.23 & 36 & 7.20 & 78 & 148 & 4.285 & 12.26 & 2.75 & 110 \\
\hline 6 & $4.05 \times 10^{6}$ & 8.650 & 9.5 & 27 & 66.60 & 23.456 & 35.18 & 31 & 7.50 & 87 & 144 & 4.571 & 12.00 & 2.75 & 104 \\
\hline $\begin{array}{l}\text { Mean } \\
\pm \text { S.E. }\end{array}$ & $\begin{array}{l}5.30 \times 10^{6} \pm \\
0.40 \times 10^{6}\end{array}$ & $\begin{array}{l}9.216 .66 \pm \\
475.76\end{array}$ & $\begin{array}{l}10.42 \pm \\
0.58\end{array}$ & $\begin{array}{l}29.50 \pm \\
3.55\end{array}$ & $\begin{array}{l}56.66 \pm \\
3.55\end{array}$ & $\begin{array}{l}20.14 \pm \\
1.67\end{array}$ & $\begin{array}{l}35.35 \pm \\
1.29\end{array}$ & $\begin{array}{l}55 \pm \\
0.93\end{array}$ & $\begin{array}{l}7.25 \pm \\
0.241\end{array}$ & $\begin{array}{l}86.50 \pm \\
2.55\end{array}$ & $\begin{array}{l}140.83 \pm \\
4.69\end{array}$ & $\begin{array}{l}4.34 \pm \\
0.167\end{array}$ & $\begin{array}{l}12.21 \pm \\
0.20\end{array}$ & $\begin{array}{l}2.75 \pm \\
0.02\end{array}$ & $\begin{array}{l}105.33 \\
6.29\end{array}$ \\
\hline
\end{tabular}


Table.5 Hematological parameters of horse before and after exercise (Mean \pm S.E)

\begin{tabular}{|c|c|c|c|c|c|c|c|c|c|c|c|c|c|c|c|}
\hline $\begin{array}{l}\text { Horse } \\
\text { Group }\end{array}$ & $\mathrm{RBC}(\mathrm{Cmm})$ & $\begin{array}{l}\text { WBC } \\
(\mathrm{Cmm})\end{array}$ & $\mid \begin{array}{l}\mathbf{H b} \\
(\mathrm{g} \%)\end{array}$ & \begin{tabular}{|l} 
PCV \\
$(\%)$
\end{tabular} & $\begin{array}{l}\text { MCV } \\
\text { cup }\end{array}$ & $\begin{array}{l}\text { MCH } \\
\text { (ppg) }\end{array}$ & $\begin{array}{l}\text { MCHC } \\
\text { percent }\end{array}$ & $\begin{array}{l}\text { ESR } \\
\mathrm{mm} / \mathbf{3 0} \\
\mathrm{mins}\end{array}$ & \begin{tabular}{|l|} 
Total \\
protein \\
$(\mathrm{g} \%)$
\end{tabular} & $\begin{array}{l}\text { Glucose } \\
(\mathrm{mg} \%)\end{array}$ & $\begin{array}{l}\mathrm{Na} \\
\mathrm{m}^{\mathrm{E}} \mathrm{q} / \mathrm{L}\end{array}$ & $\begin{array}{l}\mathrm{K} \\
\mathrm{m}^{\mathrm{E}} \mathbf{q} / \mathrm{L}\end{array}$ & $\begin{array}{l}\mathrm{Ca} \\
\left(\mathrm{mg}{ }^{\%}\right)\end{array}$ & $\begin{array}{l}\mathrm{Mg}_{2} \\
\mathrm{~m}^{\mathrm{E}} \mathbf{q} / \mathrm{L}\end{array}$ & $\begin{array}{l}\mathrm{Cl}_{2} \\
\mathrm{~m}^{\mathrm{E}} \mathrm{q} / \mathrm{L}\end{array}$ \\
\hline (before & $\begin{array}{l}5.28 \times 10^{6} \\
\pm 0.44 \times 10^{6} \\
\text { a }\end{array}$ & $\begin{array}{l}9,708.33 \\
\pm 352.40 \\
\text { a }\end{array}$ & $\begin{array}{l}10.33 \\
\pm 0.63 \\
\text { A }\end{array}$ & $\begin{array}{l}29.83 \\
\pm 1.19 \\
\mathrm{a}\end{array}$ & $\begin{array}{l}57.84 \\
\pm 3.61 \\
\mathrm{a}\end{array}$ & $\begin{array}{l}20.13 \\
\pm 1.66 \\
\mathrm{a}\end{array}$ & $\begin{array}{l}34.63 \\
\pm 1.54 \\
\mathrm{a}\end{array}$ & $\begin{array}{l}34.17 \\
\pm 0.75 \\
\mathrm{a}\end{array}$ & $\begin{array}{l}7.45 \\
\pm 0.147 \\
\text { ac }\end{array}$ & $\begin{array}{l}80.00 \\
\pm 3.87 \\
\mathrm{ac}\end{array}$ & $\begin{array}{l}144.17 \\
\pm 2.95 \\
\text { a }\end{array}$ & $\begin{array}{l}4.547 \\
\pm 0.088 \\
a\end{array}$ & & $\begin{array}{l}2.78 \\
\pm 0.033 \\
\mathrm{a}\end{array}$ & $\begin{array}{l}105.33 \\
\pm 6.12 \\
a\end{array}$ \\
\hline $\begin{array}{l}\text { (after } \\
\text { vercist }\end{array}$ & $\begin{array}{l}6.73 \times 10^{6} \\
\pm 0.43 \times 10^{6} \\
\text { b }\end{array}$ & $\begin{array}{l}11.725 .00 \\
\pm 409.54 \\
\text { a }\end{array}$ & $\begin{array}{l}11.83 \\
\pm 0.54 \\
\text { B }\end{array}$ & $\begin{array}{l}32.82 \\
\pm 1.35 \\
\mathrm{~b}\end{array}$ & $\begin{array}{l}49.55 \\
\pm 3.04 \\
\mathrm{a}\end{array}$ & $\begin{array}{l}17.94 \\
\pm 1.32 \\
\mathrm{a}\end{array}$ & $\begin{array}{l}36.05 \\
\pm 0.79 \\
\mathrm{a}\end{array}$ & $\begin{array}{l}10.33 \\
\pm 1.20 \\
\mathrm{~b}\end{array}$ & $\begin{array}{l}8.45 \pm \\
0.265 \\
\text { b }\end{array}$ & $\begin{array}{l}71.67 \pm \\
4.62 \\
\text { b }\end{array}$ & $\begin{array}{l}149.17 \pm \\
2.61 \\
\mathrm{a}\end{array}$ & $\begin{array}{l}4.51 \pm \\
0.261 \\
\mathrm{a}\end{array}$ & $\begin{array}{l}12.08 \pm \\
0.32 \\
a\end{array}$ & $\begin{array}{l}2.80 \pm \\
0.02 \\
\mathrm{a}\end{array}$ & $\begin{array}{l}102.17 \\
\pm 7.15 \\
\mathrm{a}\end{array}$ \\
\hline $\begin{array}{l}\text { 3. (4 hrs) } \\
\text { after } \\
\text { exercise }\end{array}$ & $\begin{array}{l}5.75 \times 10^{6} \\
\pm 0.56 \times 10^{6} \\
a\end{array}$ & $\begin{array}{l}9.833 .33 \\
\pm 658.10 \\
\text { a }\end{array}$ & $\begin{array}{l}10.58 \pm \\
0.65 \\
\text { A }\end{array}$ & $\begin{array}{l}29.33 \pm \\
1.43 \\
a\end{array}$ & $\begin{array}{l}52.46 \pm \\
1.29 \\
\text { a }\end{array}$ & $\begin{array}{l}19.19 \pm \\
2.18 \\
a\end{array}$ & $\begin{array}{l}36.16 \pm \\
1.85 \\
a\end{array}$ & $\begin{array}{l}25.67 \pm \\
1.14 \\
c\end{array}$ & $\begin{array}{l}7.87 \pm \\
0.27 \\
\text { ab }\end{array}$ & $\begin{array}{l}77 \pm \\
4.09 \\
\text { ab }\end{array}$ & $\begin{array}{l}142.67 \\
\pm 4.75 \\
\mathrm{a}\end{array}$ & $\begin{array}{l}4.33 \pm \\
0.196 \\
\mathrm{a}\end{array}$ & $\begin{array}{l}12.55 \pm \\
0.628 \\
a\end{array}$ & $\begin{array}{l}2.75 \pm \\
0.02 \\
\mathrm{a}\end{array}$ & $\begin{array}{l}96.83 \\
\pm 5.14 \\
\mathrm{~b}\end{array}$ \\
\hline $\begin{array}{l}\text { 4. (8 hrs) } \\
\text { after } \\
\text { exercise }\end{array}$ & $\begin{array}{l}5.30 \times 10^{6} \pm \\
0.40 \times 10^{6} \\
a\end{array}$ & $\begin{array}{l}9.216 .66 \pm \\
475.76 \\
\text { a }\end{array}$ & $\begin{array}{l}10.42 \pm \\
0.58 \\
\text { A }\end{array}$ & $\begin{array}{l}29.50 \pm \\
3.55 \\
\text { a }\end{array}$ & $\begin{array}{l}56.66 \pm \\
3.55 \\
a\end{array}$ & $\begin{array}{l}20.14 \pm \\
1.67 \\
a\end{array}$ & $\begin{array}{l}35.35 \pm \\
1.29 \\
a\end{array}$ & $\begin{array}{l}55 \pm \\
0.93 \\
\mathrm{a}\end{array}$ & $\begin{array}{l}7.25 \pm \\
0.241 \\
\text { c }\end{array}$ & $\begin{array}{l}86.50 \pm \\
2.55 \\
c\end{array}$ & $\begin{array}{l}140.83 \pm \\
4.69 \\
\text { a }\end{array}$ & $\begin{array}{l}4.34 \pm \\
0.167 \\
a\end{array}$ & $\begin{array}{l}12.21 \pm \\
0.20 \\
a\end{array}$ & $\begin{array}{l}2.75 \pm \\
0.02 \\
\mathrm{a}\end{array}$ & $\begin{array}{l}105.33 \pm \\
6.29 \\
a\end{array}$ \\
\hline
\end{tabular}

The mean glucose level of the horses in Group IV increased to $86.50 \pm 2.55 \mathrm{mg}$. percent and this was significantly $(\mathrm{P} \leq 0.05)$ higher than Group II and III (Table 5).

The mean values of serum sodium in Group I was found to be $144.17 \pm 2.95 \mathrm{mEq} . / \mathrm{L}$. Analysis of variance Indicated that the values after exercise at different intervals were not statistically significant in comparison with the control.

The mean serum potassium concentration in horses at rest was recorded to be $4.54 \pm 0.08$ $\mathrm{mEq} . / \mathrm{L}$. The potassium levels after exercise at different intervals were not significant with regard to normal value.

The mean serum calcium level of hours of Group I was estimated to be $12.10 \pm 0.24$ milligram percent. The mean values after exercise at different intervals of time were not significantly different (Table 5).

The mean serum magnesium level in Group I was recorded to be $2.78 \pm 0.03 \mathrm{mEQ} . / \mathrm{L}$. There was no significant change in the mean value after exercise.

The mean serum chloride level of the experimental animals in Group I was found to be $105.33 \pm 6.12 \mathrm{mEq} . / \mathrm{L}$. There was a significant $(\mathrm{p}<0.05)$ decrease of chloride level at 240 minutes after exercise (Table 5).

The normal mean whole blood glucose level in the experimental horses was $80.00+3.87$ $\mathrm{m}$ percent, whereas, Blood and Henderson (1981) has mentioned the normal level to be 60-100 mg./dl. Immediately after exercise a significant $(\mathrm{P} \leq 0.05)$ fall in the glucose level was observed which was in agreement with the observation of Bhatti and Shaikh (2007). The glucose level improved at 4 and 8 hours after exercise. The low value immediately following exercise was due to utilization of glucose during exercise. The stress was relieved after 4 and 8 hours of exercise and the horses had their norm al food and water. This caused a significant $(\mathrm{P} \leq 0.05)$ increase in whole blood glucose at 8 hours after exercise during which the horse had adequate rest.

The normal mean serum sodium and potassium levels in the horses were found to be within the ranges as stated by Blood and Henderson (1981). An increased sodium level immediately after exercise was recorded but the increase was not statistically significant. The levels after 4 hours and 8 hours of rest were within normal range. The serum potassium level showed a decrease after 
exercise at all the intervals of time but was not significant at any stage. The findings were not in agreement with Soliman and Nadim (1967). These workers reported that a slight decrease in sodium and a significant fall in potassium level occurred after a strenuous exercise. This might have a relation to the timing of blood collection after exercise.

The average serum calcium and magnesium levels in the horses were in the same range as mentioned by Blood and Henderson (1981). The levels in these electrolytes did not show any deviation after exercise.

The estimation of serum chloride before and soon after exercise did not show significant change. However, the mean level was significantly $(\mathrm{P} \leq 0.05)$ low at 4 hours after exercise. This could not be explained.

A complete study of the above said parameters indicated that significant $(\mathrm{P} \leq$ 0.05) increase in total erythrocyte, total hemoglobin, packed cell volume and total protein and significant $(\mathrm{P} \leq 0.05)$ fall in erythrocyte sedimentation rate and whole blood glucose occurred after exercise. There was no change in blood electrolytes. It may be concluded that analysis of blood for hemoglobin, erythrocyte sedimentation rate, total protein and whole blood glucose after exercise are indicative of stress. In any diseased condition the hemoglobin and protein mainly, may not be in normal range.

The results obtained after estimation of hematological parameters in horses after exercise showed change in some of the observations. Analysis of variance of the results was first done to find out significance of each parameter after exercise in comparison to the values before exercise. Critical differences of the parameters were thereafter recorded (Table 5) to specifically the significance of parameters at different time intervals. This helped to compare the values within the groups and at different time intervals.

The mean level of total erythrocyte in experimental horses during rest was comparatively less than those reported by Gupta et al., (2002). The low mean value was probably due to inclusion of a few old animals. Immediately following exercise there was significant $(\mathrm{P}<0.05)$ increase in the mean level (Table 5). The finding agreed with the observation of Andriichuk and Tkachenko (2015). The effect of exercise at 4 and 8 hours was not statistically significant, although it was higher. The rise in total erythrocyte soon after exercise might be due to more of cells in circulation following splenic contraction. Stimulation of hematopoietic system can lead to an increase in total erythrocyte, but this may happen due to exercise.

The average value of total leucocyte concentration in the experimental horses was within the range observed by Gopalakrishnan et al., (1973) in the Indian race horses and was comparatively less than those reported by Gupta et al., (2002). The mean level of total leucocyte showed an increase immediately after exercise but was not statistically significant. Similar results were reported by Octura et al., (2014). The level dropped at 4 and 8 hours after exercise reaching the reaching the range of the control group (Table 5 ). Increased blood cell number due to splenic contraction might have attributed to increase in total leucocyte level during exercise, though it was not significant.

The mean hemoglobin level of the horses during rest was less than that reported by earlier workers by Octura et al., (2014) and Andriichuk and Tkachenko (2015). Similar low value was also obtained in total erythrocyte level in these horses before exercise. The reason for a low value may be 
the inclusion of more number of old horses, which presented lot of variation in confirmation and breed character. Low hemoglobin was directly proportional to low erythrocyte level. Immediately following exercise there was an increase in hemoglobin concentration. This was related to a similar increase in erythrocyte count after exercise. Four and eight hours after exercise the hemoglobin dropped to normal range as was in control group. Identical observation was also noted in total erythrocyte level.

The average level of packed cell volume of the horses was lower than the levels reported by Gopalakrishnan et al., (1973) Fregin (1980) and Blood and Hendersen (1981), Such low value was evident as the total erythrocyte level was also lower in the horses. However, an increase $(\mathrm{P}<0.01)$ in level was recorded in the horses after exercise. Similar results were also obtained by Kosslla (1976) and Fregin (1980). The significant increase in the packed cell volume soon after exercise was due to significant increase in erythrocyte count (Table 5) and loss of fluid during exercise through sweating. The average values reached the control level at 4 and 8 hours after exercise. A significant increase in packed cell volume in horses immediately after exercise denoted hemoconcentration during increased packed cell volume results from hemoconcentration following exercise and due to release of more number of erythrocyte from spleen.

These parameters were calculated using the erythrocyte and hemoglobin values. A decrease in $\mathrm{MCV}$ and $\mathrm{MCH}$ and an increase in $\mathrm{MCHC}$ has been observed immediately after exercise but none of the values were statistically significant. Reduced mean corpuscular volume is probably the result of shrinkage of red cell wall resulting from changed osmotic pressure, thus, there were more cells in a comparatively lesser PCV.
Low $\mathrm{MCH}$ was also related to the above factor. The corpuscular hemoglobin concentration was higher than the control level but was not significant though the whole blood hemoglobin concentration was significantly $(\mathrm{P}<0.05)$ higher than the control value immediately after exercise.

The normal erythrocyte sedimentation rate in the horses was near the range reported by Octura et al., (2014). The sedimentation rate was significantly $(\mathrm{P}<0.05)$ low after exercise (Table 5). Such condition was due to increased muscular action and respiratory rate during exercise leading to quicker movement of circulating blood in the tissues. It was also observed that though the sedimentation rate increased after 4 hours of exercise, yet it was significantly $(\mathrm{P}<0.05)$ lower than the control level (Table 5). Further, it has also been recorded that though the mean erythrocyte level increased significantly $(\mathrm{P}<0.05)$ less at 4 hours after exercise. The sedimentation rate reached the normal level after 8 hours of exercise

In the present experiment the horses were allowed to trot for 30 minutes and at the end of exercise blood sample were collected to estimate the erythrocyte sedimentation rate. ESR during rest after exercise was $34.17+$ 0.75 and $10.33+1.20 \mathrm{mn} / 30$ minutes. Such a low erythrocyte sedimentation rate was not observed in this experiment even though the horses were given 30 minutes of trotting.

The normal mean serum total protein value of the experimental horses was higher than that reported by Anderson et al., (1975) significant $(\mathrm{P}<0.01)$ increase after exercise was observed. Critical difference further revealed that the total protein level was not significantly high at 4 hours after exercise than the control and was not significantly low than the level immediately after exercise. These changes in total protein indicated that 
exertion and less water intake might have caused significantly $(\mathrm{P}<0.05)$ high total protein level following exercise. Two hours after exercise all the horses were supplied with their normal ration.

It can be concluded that the horses maintained under hot and humid climatic conditions of Orissa exhibited similar changes in blood when given exercise and the changes were of transitory nature. Further study is needed to be taken up to ascertain the facts responsible for a low erythrocyte count in these horses.

\section{Acknowledgements}

The authors are grateful to the Dean, College of Veterinary Science and Animal Husbandry, OUAT, Bhubaneswar and Commandant, 1 (Orissa) R \& V Sqn., N.C.C for providing necessary facilities in the Department of Livestock Production and Management and N.C.C. division respectively for smooth conduction and completion of the research within the stipulated time.

\section{References}

Aguilera-Tejero, E., Estepa, J.C., Lopez, I., Bas, S., Mayer-Valor, R. and Rodriguez, M. (2000). Quantitative analysis of acid-base balance in show jumpers before and after exercise. Res Vet Sci., 68:103-8.

Anderson, M.G. (1975). The influence of exercise on serum levels in the horse. Equine Veterinary Journal., 7(3):160-5.

Andriichuk, A. and Tkachenko, H. (2015). Seasonal variations of hematological indices in equines involved in recreational horse riding. Journal of Ecology and Protection of the Coastline., 19: 11-12.

Barrey, E., Valette, J. P. (1993). Exerciserelated parameters of horses competing in show jumping events ranging from a regional to an international level. Annual Zootech., 42: 89-98.
Bhatti, R. and Shaikh, D.M. (2007).The Effect of Exercise on Blood Parameters. Pakistan Journal of Physiology., 3(2): 4446.

Blood, D.C., and Hendersen, J.A. (1981). Veterinary Medicine $5^{\text {th }}$ ed., Williams and Wilkin Company, Baltimore.

Burtis, C.A and Ashwood, E.R. (1999). eds. Tietz Textbook of Clinical Chemistry. WB Saunders, Philadelphia, PA, pp. 617721.

Coles, E.H. (1974). Veterinary Clinical Pathology. W.B.S. Saunders Co. Philadelphia, P.A.

De Miranda, R. L., Mundim, A.V., Silveira Saqui, A.C., Souza Costa, A., Guimaraes, E. C. Goncalves, F. C., Ozanam Carneiro, F. and Silva, E. (2009): Biochemical serum profi le of equine subjected to team penning. Vet. Clin. Pathol., 18: 313-319.

Frape, D. (2010). Water requirements and fl uid losses. In: Equine Nutrition and Feeding, Wiley Blackwell Publications, pp. 37-45.

Fregin, G.F. (1980). General discussion of physiological observations recorded on 117 horses during 100 mile endurance ride. In : Proceeding of the Annual convention.

Gopalakrishnan, L.V., Viswanathan, S. and Bhaskar, C.G. (1973). Plasma inorganic Calcium and Phosphorus in Indian horses. Indian Vet. J., 50: 308-311.

Gupta, A. K. Sanjay Kumar and Yash Pal. (2012). Biochemical, Haematological and Thyroid Hormone Profile in Healthy Indian Kathiawari Horses. Asian-Aust. J. Anim. Sci., 15(8): 1215-1221.

Jain, N. C. (1986). Schalm's Veterinary haematology, $4^{\text {th }}$ edn. Lea and febiger, Philadelphia.

Johnson, A.M., Rohlfs, E.M. and Silverman, L.M. (1999). Proteins. In: C.A. Burtis and E.R. Ashwood, editors. Tietz Textbook of Clinic. Chem. $3^{\text {rd }}$ ed. W.B. Saunders Co. Philadelphia. pp. 477-540.

Kosslla, V., Tanhuanpaa, E., Peltonen, T. and Vartanen, E. (1976). Effect of physical stress on the pulse and respiration rate and blood composition of riding horses 
and trotters. Annales Agricultura Fenniae, 15: 322-329. Quoted from Vet. Bull., 48: 1274.

Lindinger, M. I. and Heingenhauser, G. J. F. (2008). Last word on point: counterpoint: lactate is not the only physicochemical contributor to the acidosis of exercise. $J$. Appl. Physiol., 105: 369.

Mukai, K., Takahashi, T., Eto, D., Ohmura, H., Tsubone, H. and Hiraga, A. (2007). Heart rates and blood lactate response in Thoroughbreds horses during a race. $J$ Equine Sci., 18:153-60.

Munoz, A., C. Riber, P. Trigo, F. M. Castejón (2010). Dehydration, electrolyte imbalances and renin-angiotensinaldosterone-vasopressin axis in successful and unsuccessful endurance horses. Equine Vet. J. 42, 83-90.

Munoz, A., Cuesta, I., Riber, C., Gata, J., Trigo, P. and Castejon, F.M. (2006). Trot asymmetry in relation to physical performance and metabolism in equine endurance rides. Equine Vet J., 36:50-4.

Octura, J. E.R., Lee, K. J., Cho, H.W., Vega R. S.A., Choi,J.Y., Jeong-Woong Park, Teak-Soon Shin, Seong-Keun Cho, Byeong- Wook Cho, W. and Byung. (2014). Elevation of Blood Creatine Kinase and Selected Blood Parameters after Exercise in Thoroughbred Racehorses (Equus caballus L.). Journal of Research in Agriculture and Animal Science., 2(5):07-13.

Piccione, G., Casella, S. Giannetto, C., Monteverde, V. and Ferrantelli, V. (2009). Exercise-induced modifications on haematochemical and electrophoretic parameters during 1600 and 2000 meters trot races in standardbred horses. J. Appl. Anim. Res., 35: 131-135.
Robert, C., Goachet, A., Fraipont, A., Votion, D.M., Van Erck, E. and Leclerc, J.L. (2010). Hydration and electrolyte balance in horses during an endurance season. Equine Vet.J., 42: 98-104.

Santos, S.A., Silva, R.A., Azevedo, J.R., Mello, M.A., Soares, A.C. and Sibuya, C.Y. (2001). Serum electrolyte and total protein alterations in Pantaneiro horse during long distance exercise. Arq Bras Med Vet Zootec., 53:351-7.

Snedecor, G.W. and Cochran, W.G. (1989). Statistical methods, $8^{\text {th }}$ edition. Lowa State University Press, Ames, Iowa.

Soliman, M.K. and Nadim, M.A. (1967). Calcium Sodium and Potassium level in the serum and sweat of healthy horses after strenuous exercise. Zentbl. Vet. Med., 14A: 53-56. Quoted from Vet. Bull., 37: 2872.

Tateo, A., Valle, E., Padalino, B., Centoducati, P. and Bergero, D. (2008). Change in some physiologic variables induced by Italian traditional conditioning in standard bred yearling. J. Equine Vet. Sci., 28: 743-750.

Teixiera-Neto, A.R., Ferraz, G.C., Moscardini, A.R., Balsamao, G.M., Souza, J.C. and Queiroz-Neto, A. (2008). Alterations in muscular enzymes of horses competing long-distance endurance rides under tropical climate. Arq Bras Med Vet Zootec., 60:543-9.

Van den berg, M. (2009). Exercising horses in summer time, sweating and electrolyte losses. Horses and people, pp. 6-8.

Warwick, B. (2004). Foreword. In: Equine Sports Medicine and Surgery. Basic and Clinical Sciences of Equine Athlete. (Hinchcliff, K. W., A. J. Kaneps, R. J. Geor, Eds.), Sauders Press, China.

\section{How to cite this article:}

Kanungo, S., C.R. Pradhan, L.K. Babu, K. Behera, A.K. Palei, B. Jena and Das, D.P. 2018. Effect of Periodicity of Exercise on Serum Metabolites of Stall Housed competition Horses under Climatic Conditions of Odisha, India. Int.J.Curr.Microbiol.App.Sci. 7(03): 3338-3347. doi: https://doi.org/10.20546/ijcmas.2018.703.385 